\title{
Cosmic Plasmas and Particle Acceleration: An Introduction
}

\author{
André Balogh • Andrei Bykov • Robert Lin • \\ John Raymond • Manfred Scholer
}

Published online: 13 October 2012

(C) Springer Science+Business Media Dordrecht 2012

\section{Background: From the Magnetosphere to Cosmic Plasmas}

The topic of this volume, Particle Acceleration in Cosmic Plasmas, is the first in a series of three broad topics, each intended for a volume in Space Science Reviews and the Space Science Series of ISSI, that cover aspects of astrophysical plasma phenomena on all scales, from the Earth's magnetosphere to the scale of clusters of galaxies. The other two topics cover "The Microphysics of Cosmic Plasmas" and "Structure Formation and Dynamics in Cosmic Plasmas". The motivation for the series can be found in the conclusions of a discussion Forum held in the International Space Science Institute, Bern, Switzerland, in March 2009. The topic of the Forum was the question "Is there a future for magnetospheric research?", originally formulated at a meeting of COSPAR's Scientific Advisory Committee in March 2008.

The discovery, exploration and detailed understanding of the Earth's magnetosphere have been key scientific goals and the focus of significant activities in scientific space research in

\footnotetext{
A. Balogh $(\bowtie)$

Imperial College London, Prince Consort Road, London SW7 2AZ, UK

e-mail: a.balogh@imperial.ac.uk
}

\author{
A. Bykov \\ Ioffe Institute of Physics and Technology, Russian Academy of Sciences, Politechnicheskaya, 26, \\ St. Petersburg, 194021, Russia \\ R. Lin \\ Department of Physics, University of California Berkeley, 366 LeConte Hall 7300, Berkeley, \\ CA 94720-7300, USA \\ J. Raymond \\ Harvard-Smithsonian Center for Astrophysics, 60 Garden Street, Cambridge, MA 02138, USA \\ M. Scholer \\ Max-Planck-Institute for Extraterrestrial Physics, Giessenbachstrasse, 85748 Garching, Germany
}


the past fifty years. In terms of resources devoted to magnetospheric research and the numbers of scientists that have been directly engaged in it, the discipline dominated the early part of the space age. In the past thirty years or so, other scientific disciplines have progressed to match or surpass the activity levels in magnetospheric research. Nevertheless, the topic remains very active, although in parallel with other scientific areas, the sophistication of the missions that provide much of the raw material for the research has increased significantly.

The Earth's magnetosphere contains long-lived plasmas with well-separated scales and exhibits examples of key universal processes that are amenable to in-situ as well as to remote-sensing, global observations. This makes it a unique plasma physics laboratory, one that is recognised increasingly as one of the tools of wider scientific research interests, both on the large, astrophysical scale and the small, Earth-based laboratory scale. In addition, the magnetosphere is our immediate environment in the larger Sun-Earth system; the effects of the dynamics of this system are relevant to the whole range of applied activities in space and require secure forecasting of conditions in the magnetosphere.

Magnetospheric research is thus a particularly successful branch of space plasma physics. The physics of plasmas in the Earth's magnetosphere has been extended to the other five magnetised planets in the solar system: Mercury, Jupiter, Saturn, Uranus and Neptune. There are of course strong similarities in the way the different planets' magnetic fields form the planet-centred, plasma-filled magnetic bubbles through their interaction with the solar wind, but there are also significant differences that have allowed the exploration of different parameter ranges in magnetospheric physics.

While the overall picture as how plasma processes work in the magnetosphere is generally known, thanks to the observations gathered during the past decades, much of the basic physics at the micro-scale still remains unclear, as well as how the various processes interact. The future thrust of magnetospheric research should thus be primarily to understand the microphysics and its coupling to meso- and macro-scales. This represents a natural evolution of the subject and allows the links to other areas of research that are essential for the continued relevance of the research and can justify the resources devoted to it.

The origins of research in space plasma physics are difficult to identify precisely. Several strands of apparently independent research topics, in near-Earth space, in the solar atmosphere and in a variety of astrophysical environments have identified over several decades the all-pervading importance of plasmas in the Universe. The fully or partially ionised gases, often at very high temperatures, are often the key constituents of astrophysical objects and their broader environment. The physics of space, be it interstellar, interplanetary or planetary is largely synonymous with the physics of plasmas, albeit described by parameters that cover many orders of magnitudes. The different contexts in which plasma physics has evolved have led to often different technical terms and different descriptions with, generally speaking, little overlap in insights due to the vast differences in spatial scales and material parameters.

There are also, however, important plasma parameters which overlap (in the order of magnitude sense) between very different environments. Such are the plasma beta (the ratio of energy densities in the plasma particles and the magnetic field), the Alfvén Mach number (the ratio of the plasma flow speed to the velocity of hydrodynamic waves-the Alfvén speed) and the Magnetic Reynolds Number (a dimensionless parameter that describes the relative importance of magnetic advection and diffusivity). The objectives of reviewing jointly space plasma physics on all cosmic scales include the identification and quantification of both large differences and overlapping parameter regimes.

As one of the outcomes of the ISSI Forum on the future of magnetospheric research, a series of three Workshops was proposed on the exploration of phenomena and processes in 
cosmic plasmas on all scales and on reviewing current knowledge and outstanding questions in the different plasma regimes. The argument for joining the topics of plasma physics research across the magnetopsheric, solar and astrophysical research communities was to initiate and develop communication channels to generate a multi-scale view that would bring under one roof and between the same covers the experience from the diverse communities.

\section{Objectives of the ISSI Workshop on Particle Acceleration in Cosmic Plasmas}

The first ISSI Workshop in the series on cosmic plasmas, on particle acceleration, was held on 16 to 20 May 2011. As is always the case with ISSI Workshops, the printed outcome as presented in this volume is not the proceedings of the Workshop, but a series of review papers which were conceived during the Workshop, benefiting from the direct interaction and collaboration of experts covering the broad range of topics in cosmic plasmas. This volume is the outcome of that first Workshop.

The overall objective of the Workshop was the critical assessment of acceleration mechanisms and observations on a range of scales from suprathermal particles in the heliosphere to high energy cosmic rays $\left(10^{3} \mathrm{eV}\right.$ to $\left.10^{20} \mathrm{eV}\right)$ and a constructive comparison of acceleration processes across the scales.

Particle acceleration is a ubiquitous process in cosmic plasmas. While plasma heating occurs also in all environments (when Maxwellian particle distributions are generated), the generation of non-thermal energetic particles is an important factor in the energy conversion processes that take place in plasmas. As listed below, there are many processes in astrophysical plasmas that lead to energetic particles. However, there important questions concerning the acceleration of non-thermal particles. One concerns the apparent efficiency of acceleration processes; in particle acceleration sites, a very significant fraction of the available energy is converted to energetic particles, illustrated, for instance, by the tens of percent of the energy of larger solar flares to appear in the form of energetic protons an electrons. This efficiency demonstrates clearly that the acceleration of energetic particles is not a by-product of high-energy phenomena in plasmas, but an integral, even critical part of them. Another question concerns the very high energies that accelerated particles can reach. Again, in the more energetic solar flares, particles can be accelerated to $\mathrm{GeV}$ energies by mechanisms that are as yet not clearly understood. The energy spectrum of cosmic rays detected at Earth reaches up to $10^{21} \mathrm{eV}$, implying very efficient, sustained acceleration mechanisms on cosmic scales, and also implying physical processes that involve black hole rotation and dark matter. Cosmic rays have a significant energy density in interstellar space, of order $1 \mathrm{eV} / \mathrm{cm}^{3}$, several times the energy density of starlight photons. Whether in the Earth's magnetosphere or in the environment of distant astrophysical objects, the acceleration of energetic particles is a key question that needs answers to understand not only cosmic plasmas, but the objects themselves.

The volume covers the following main themes:

- Review of observations of accelerated particles on all scales: in situ and remotely from space, and also ground-based, from the magnetosphere to extragalactic scales

- A detailed assessment of the physical processes underlying particle acceleration in the different physical environments: the aurorae, the Earth's radiation belts, bow shock- and interplanetary shock associated particles, flare-accelerated and generally solar energetic particles, acceleration at and beyond the heliospheric termination shock, particles accelerated by supernova remnants, jets, pulsars, gamma ray bursts. 
- A critical evaluation of the contribution of competing processes in different physical contexts.

- Acceleration mechanisms in general:

- Shocks and diffusive shock acceleration, magnetic field amplification

- Stochastic acceleration (2nd order Fermi): its mechanisms in the different environments, and its possible role on largest scales for accelerating highest energy cosmic rays

- Turbulent acceleration: the description of mechanism and applicability across the cosmic scales

- Acceleration in current sheets; acceleration by parallel electric fields; their applicability in different physical contexts

- Nature of acceleration process and its possible scale-dependence that gives a distribution function with a power law in particle speed, with a spectral index of -5

- The contribution of different simulation and modelling studies to the understanding of the particle acceleration processes

- The status of particle acceleration research and future perspectives: identification of shortcomings in the theory, gaps in the observations and the improvements needed to remedy those problems.

Acknowledgements The Editors wish to express their indebtedness to the International Space Science Institute and its staff for creating the special atmosphere in which this Workshop could be held, to generate lively discussions among a very diverse set of space plasma physicists, solar physicists and astrophysicists that have led to the review articles in this volume. In particular, our thanks are due to ISSI's Executive Director, Roger Bonnet and his fellow directors who approved the series of Workshops on Cosmic Plasmas, on the recommendation of ISSI's Science Committee. The Workshop could not be successful without the direct assistance of ISSI's staff, in particular Jennifer Zaugg, Saliba Saliba and Maurizio Fallanga. The Editors thank the contributors to this volume and also the reviewers who helped in achieving a consistently high standard of presentation and content. Thanks are also due to the staff of Springer, Space Science Reviews and their typesetters who successfully ensured the high quality of the finished product. 